\title{
Finite element analysis of multi-piece post-crown restoration using different types of adhesives
}

\author{
Lin-Wei Lü ${ }^{1}$, Guang-Wei Meng ${ }^{1}$ and Zhi-Hui Liu ${ }^{2}$
}

The multi-piece post-crown technique is more effective in restoring residual root than other restoration techniques. Various types of adhesives have different material properties that affect restoration. Therefore, the choice of adhesive is particularly important for patients. However, the effect of different kinds of adhesives was not too precise by experimental methods when concerning about individual differences of teeth. One tooth root can only be restored with one type of adhesive in experiment. After the mechanical test, this tooth root cannot be restored with other adhesives. With the help of medical imaging technology, reverse engineering and finite element analysis, a molar model can be reconstructed precisely and restored using different types of adhesives. The same occlusal and chewing loads were exerted on the same restored residual root models with different types of adhesives separately. Results of von Mises stress analysis showed that the adhesives with low Young's modulus can protect the root canal effectively. However, a root canal concentration is apparently produced around the root canal orifice when chewing. Adhesives with large Young's modulus can buffer the stress concentration of the root canal orifice. However, the root canal tissue may be destroyed because the adhesive is too hard to buffer the load.

International Journal of Oral Science (2013) 5, 162-166; doi:10.1038/ijos.2013.50; published online 26 July 2013

Keywords: adhesive; finite element analysis; molar restoration; multi-piece post-crown; stomatology

\section{INTRODUCTION}

Tooth loss caused by decayed tooth or external force damage is a common, frequently occurring disease. Although the crown is missing, the residual root still remains in the alveolar bone of most patients. Residual root can be effectively preserved through a successful root canal therapy. The main method of fixing residual root is post-core restoration solution. General and multi-piece post-crown methods are two basic forms of post-core restoration solution. If a general postcrown method is applied on the molars, the post cannot be assembled into the root canal because the curvature of a molar root is significantly larger than that of the other roots and the angle between two molar root canals of one molar is too wide. Forcible assembly can easily crack the root wall tissues. Multi-piece post-crown restoration can fix this problem. ${ }^{1}$ The crown and the post are two separate parts that are fixed using adhesives. The post and the root canal can be matched accurately using this method. In the multi-piece post-crown restoration technique, the crown and the post are cast into one so that the crown can be fixed stably. However, restoration means a change in the natural biomechanical balance of the tooth. The Young's modulus of the materials involved can vary significantly and does not fully match that of natural teeth. ${ }^{2}$ Cyclical (fatigue) loading can disorganize the coherence of the restoration. ${ }^{3-6}$ The load applied to the crown is transmitted to the root through a post. The root bears most of the load and is easily split. ${ }^{7}$ Therefore, a stable retention is necessary and the adhesive becomes a buffer, making the choice of adhesive particularly important. $^{8}$
Some scholars used experimental method to study the retention force on the dental prosthesis of different kinds of adhesives. However, different kinds of adhesives cannot be applied on the same tooth, and the stress distribution cannot be measured between root and prosthesis. ${ }^{9}$ Three-dimensional finite element analysis has its unique advantages, such as repeatability, high accuracy and efficiency. Different kinds of adhesives with the same form and different loads can be applied on the same tooth. Aside from the ability to measure the stress state at any point and interface, the deformation of the models can also be calculated, which can provide the data reference for the stability of the restoration solution. ${ }^{10}$ All these advantages were not realized during the experiments because the sizes of teeth were too small to measure and the internal stress distribution of restored teeth cannot be measured.

Numerous scholars analyzed mechanical properties of the restored teeth using finite element method. ${ }^{11-13}$ However, Maceri merely fitted the prosthesis with simple geometries and classified the material properties at the outermost grid of restoration models as adhesives. Without an independent complex adhesive model, contact relationships cannot be set and stress and displacement distributions of adhesive cannot be obtained. Ausiello et al. ${ }^{12}$ used a spring element instead of the original adhesive models. However, springs could not simulate the form of an adhesive, indicating that springs could not simulate the mechanical properties of adhesive.

In this article, we selected one patient with low occlusion-gingiva space root to undergo multi-piece post-crown restoration. A part of

\footnotetext{
${ }^{1}$ Department of Engineering Mechanics, Jilin University, Changchun, China and ${ }^{2}$ Hospital of Stomatology, Jilin University, Changchun, China Correspondence: Professor ZH Liu, Hospital of Stomatology, Jilin University, No. 1500 Qinghua Road, Changchun 130022, China 
the residual root was scanned through computed tomography (CT). Finite element models can be established using CT images by reverse engineering with MIMICS software. We obtained the analysis results of various kinds of adhesives under different loads. The type of adhesive that most suitable for multi-pieces post-crown restoration can be determined after a statistical analysis. This study provided basic data references for clinical application to achieve the best therapeutic effect.

\section{MATERIALS AND METHODS}

\section{Establishment of residual root model}

A mandibular first molar in vitro with no dental disease was selected to excise the crown at the edge of the dentino-enamel junction. Root canal therapy and preparation for dental prosthesis were performed on the following day. The residual root area of the first molar was CTscanned. The Philips 256 spiral CT scanning device (Philips, Amsterdam, Netherlands) was provided by No. 2 Hospital of Jilin University. CT scanning acquisition was performed with $120 \mathrm{kV}$, $351.17 \mathrm{mAs}, 1024 \times 1024$ matrix, $0.024 \mathrm{~mm}$ pixel size and $0.333 \mathrm{~mm}$ increment. All CT images were imported into reverse engineering with MIMICS software (Materialise Inc., Leuven, Belgium) for threedimensional reconstruction. After a series of operations, that is, creating masks, mask editing, region growing, calculating threedimensional objects and remeshing, the residual root model of first molar was established, as illustrated in Figure 1a.

\section{Establishment and assembly of multi-piece post-crown models}

The prosthesis had two parts; first was the post and crown, then a nail is inserted into the other root canal. The prosthesis was also CT-scanned, with scanning parameters similar to that of a residual root. The CT data from the first part were imported into MIMICS for three-dimensional reconstruction. As the size of the nail was too small, a large error occurred during the scanning. Thus, we measured the nail size by micrometer and created the model using MAGICS 13.0 (Materialise Inc., Leuven, Belgium). The prosthesis models are shown in Figure 1b.

A part of the alveolar model was established to simulate the constraints on the root. A box model was created using MAGICS, and a Boolean subtraction operation was conducted between the box model and residual root. Hence, the alveolar model was established. In a clinical treatment, the root and prosthesis were fixed using adhesives. Thus, we imported the prosthesis models into MAGICS and offset the post-crown model outside with $0.1 \mathrm{~mm}$. A Boolean subtraction operation between the offset model and the original model was conducted. The resulting model was the adhesives. Using the same method, the adhesive around the nail can also be conducted. The adhesive models are shown in Figure 1c, while the assembled models are shown in Figure 1d.

All models were imported into Abaqus 6.11 (SIMULIA Inc., Providence, RI, USA), finite element analysis software, to generate finite element models with four-node tetrahedron elements. Information on the nodes and elements in all models are shown in Table 1.

\section{Definition of material properties}

The material property of the root was inhomogeneous. The relationship between the gray value of CT images and apparent bone density was nearly linear, and apparent bone density was correlated with Young's modulus. In this study, we divided the gray value of the CT images into 200 pieces to obtain 200 materials. The empirical expressions from the MIMICS software references were chosen as the Young's modulus of the teeth and were expressed as follows: a

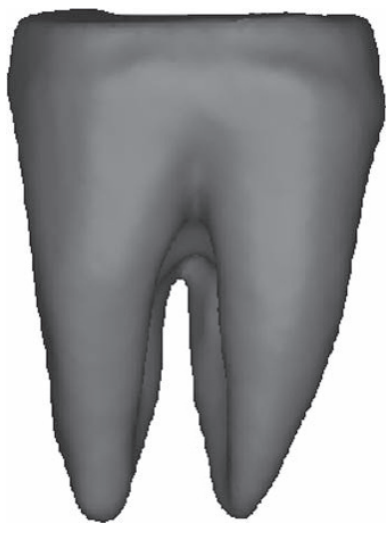

b

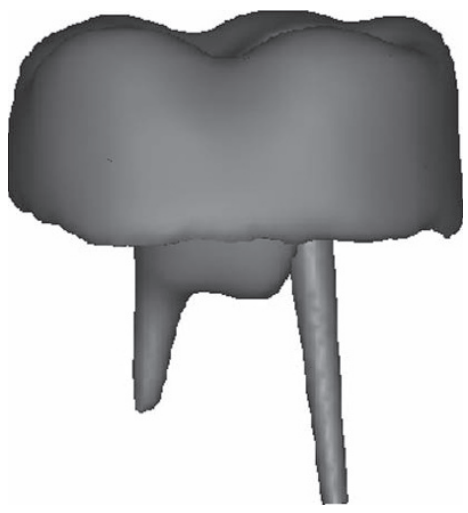

d

C
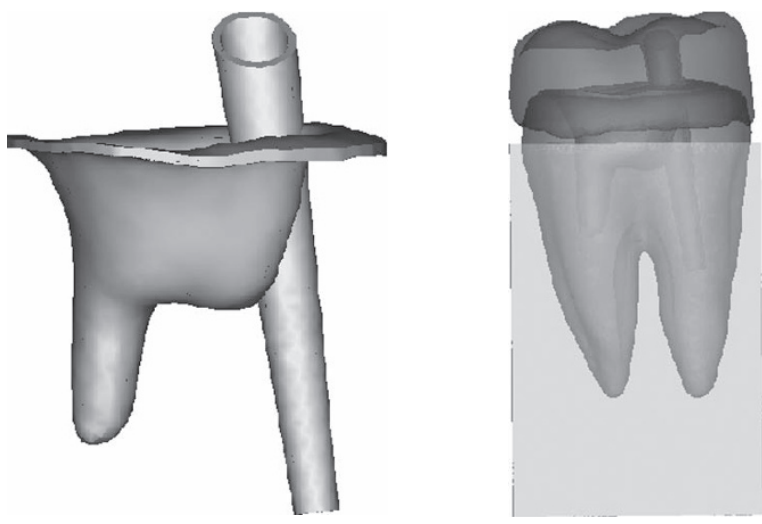

Figure 1 Establishment of finite element models in multi-piece post-crown restoration. (a) Residual root model of the mandibular first molar; (b) multi-piece post-crown prosthesis models of the first molar; (c) adhesive models of multi-piece post-crown prosthesis; (d) assembled models of multi-piece post-crown restoration.

$$
\begin{gathered}
\rho=1.067 \times \mathrm{HU}+131\left(\mathrm{~g} \cdot \mathrm{cm}^{-3}\right) \\
E=0.09882 \times \rho^{1.56}(\mathrm{MPa})
\end{gathered}
$$

where HU represents the gray value of the CT images with the unit of Hounsfield and $\rho$ represents the densities of teeth with the unit of $\mathrm{g} \cdot \mathrm{cm}^{-3}$. The material property of the prosthesis was defined as titanium and the material property of alveolar is defined as cancellous bone. Five types of adhesives were selected for analysis. They were zinc phosphate, carboxylic acid zinc, glass ionomer, panavia F and Superbond.

Table 1 Information on nodes and elements of all finite element models

\begin{tabular}{lrr}
\hline Models & Nodes & Elements \\
\hline Alveolar bone & 123784 & 671573 \\
Root & 48545 & 260310 \\
Crown & 28239 & 145825 \\
Nail & 1424 & 5985 \\
Adhesive & 5552 & 17219 \\
\hline
\end{tabular}



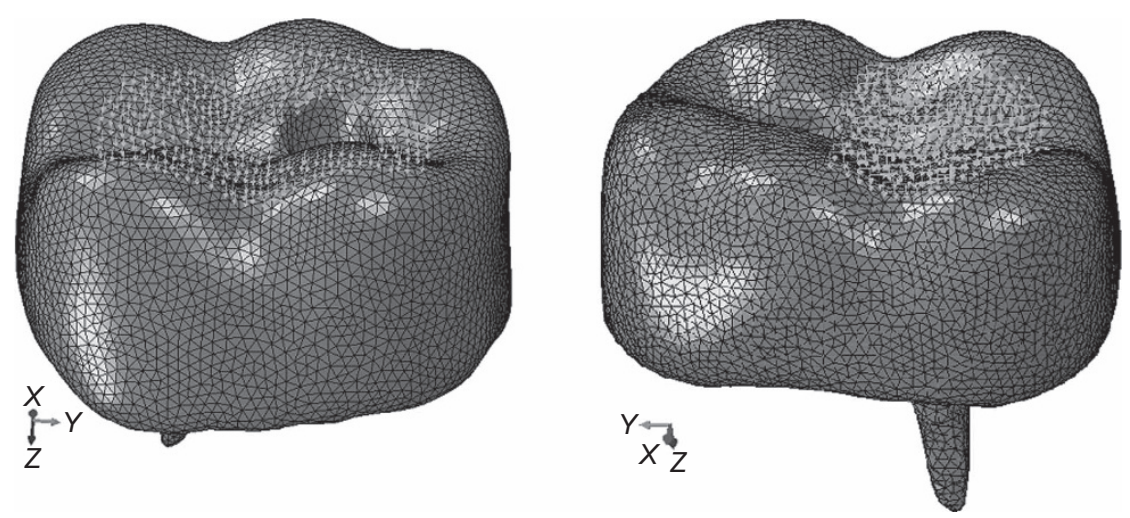

Figure 2 Points and directions of loads. (a) Points and directions of loads in biting condition; (b) points and directions of loads in chewing condition.

Table 2 Material properties of the models

\begin{tabular}{lcc}
\hline Materials & Young's modulus/GPa & Poisson's ratio \\
\hline Zinc phosphate & 22.4 & 0.35 \\
Carboxylic acid zinc & 5.11 & 0.30 \\
Glass ionomer & 4 & 0.35 \\
Panavia F & 18.3 & 0.33 \\
Superbond & 1.8 & 0.25 \\
Alveolar & 13.7 & 0.30 \\
Prosthesis & 78 & 0.30 \\
\hline
\end{tabular}

The Young's modulus and poison ratio of these adhesives are listed in Table 2. ${ }^{14}$

\section{Definition of interaction relationships}

The interaction relationship between the root and alveolar was defined as tie. All prostheses were fixed on the root. Therefore, the interaction relationships between the adhesives and the other objects were tie. However, the crown was significantly in close contact with the root, with only a small amount of adhesive between them. We defined the interaction relationship between the crown and root as friction. The coefficient of friction was 0.3 .

\section{Loading and boundary condition}

The bottom of the alveolar was rigidly fixed to simulate the constraint from the alveolar bone. A $300 \mathrm{~N}$ force was exerted on the occlusal surface of the crown to simulate occlusion. The direction of the load was perpendicular to the surface of the crown. The area of the load and direction of the force are shown in Figure 2a. Another $300 \mathrm{~N}$ force was exerted on the occlusal surface of the crown to simulate chewing. The load was separated into two directions. Direction A was perpendicular to the surface of the crown, while direction B was parallel to the surface of the crown to simulate the shear force during the chewing process. The points of the load and direction of the force are shown in Figure 2b.

\section{RESULTS}

Figure 3 shows the stress nephogram of the first molar restored with the adhesive of zinc phosphate. The stress concentration areas were determined clearly in biting and chewing conditions. In the biting condition, stress concentration occurred near the root canal and at the bottom of the root canal. Stress concentration only happened near the root canal in the chewing condition, and the maximum stress was much larger than in the biting condition. The maximum von Mises stresses of the root canal with different adhesives are shown in Table 3, Rows 1 and 2. These two rows show that with the increase of the Young's modulus of the adhesives, the maximum stress of the root canal also increased. The Young's modulus of zinc phosphate was the largest. Thus, the stress-shielding effect produced by the adhesive was the smallest and the stress exerted on the root canal was the largest. The Young's modulus of Superbond C\&B was the smallest. Hence, the stress-shielding effect produced by the adhesive was the largest and the stress exerted on the root canal was the smallest. In chewing, the root must bear the pressure exerted on it as well as the load perpendicular to it. Table 3 shows that the load exerted on the root canal in chewing was significantly larger than that in biting. The maximum stress in the biting condition was similar with Li's result, ${ }^{14}$ which only include the biting condition. The maximum stress in the chewing condition was larger than in the biting condition. Therefore, the chewing condition is more important when considering the load exerted on the molar.

The maximum von Mises stresses at the contact area between the root canal orifice and the crown are shown in Table 3, Rows 3 and 4. In the biting condition, the load exerted on the contact surface between crown and root edge was even. In the chewing condition, the shear force exerted on the crown resulted in the force area becoming a part of the contact surface. The maximum stress of the root edge was larger than that in the biting condition. The result showed that the maximum stress was smallest at the root edge using zinc phosphate and largest at the root edge using superbond. The stress cloud charts show that significant stress concentration was produced in the chewing condition. Thus, in the course of long-term use, cracks begin at the stress concentration points.

Table 3 Maximum von Mises stress inside the root canal with different adhesives and maximum von Mises stress at the root canal orifice /MPa

\begin{tabular}{|c|c|c|c|c|c|}
\hline Loading conditions and positions & Superbond $C \& B$ & Glass ionomer & Carboxylic acid zinc & Panavia F & Zinc phosphate \\
\hline Bite (root canal) & 10.2441 & 14.3095 & 14.8152 & 19.268 & 19.2837 \\
\hline Chewing (root canal) & 19.8013 & 23.3697 & 24.6665 & 28.0305 & 28.2582 \\
\hline Bite (root canal orifice) & 27.08 & 23.37 & 23.00 & 22.22 & 22.22 \\
\hline
\end{tabular}


a
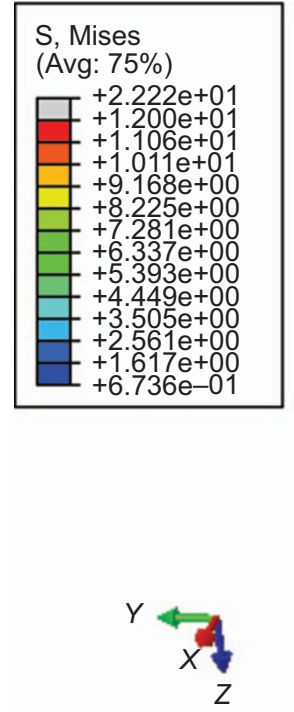

C
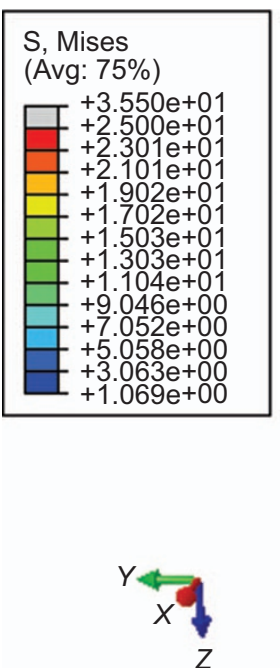
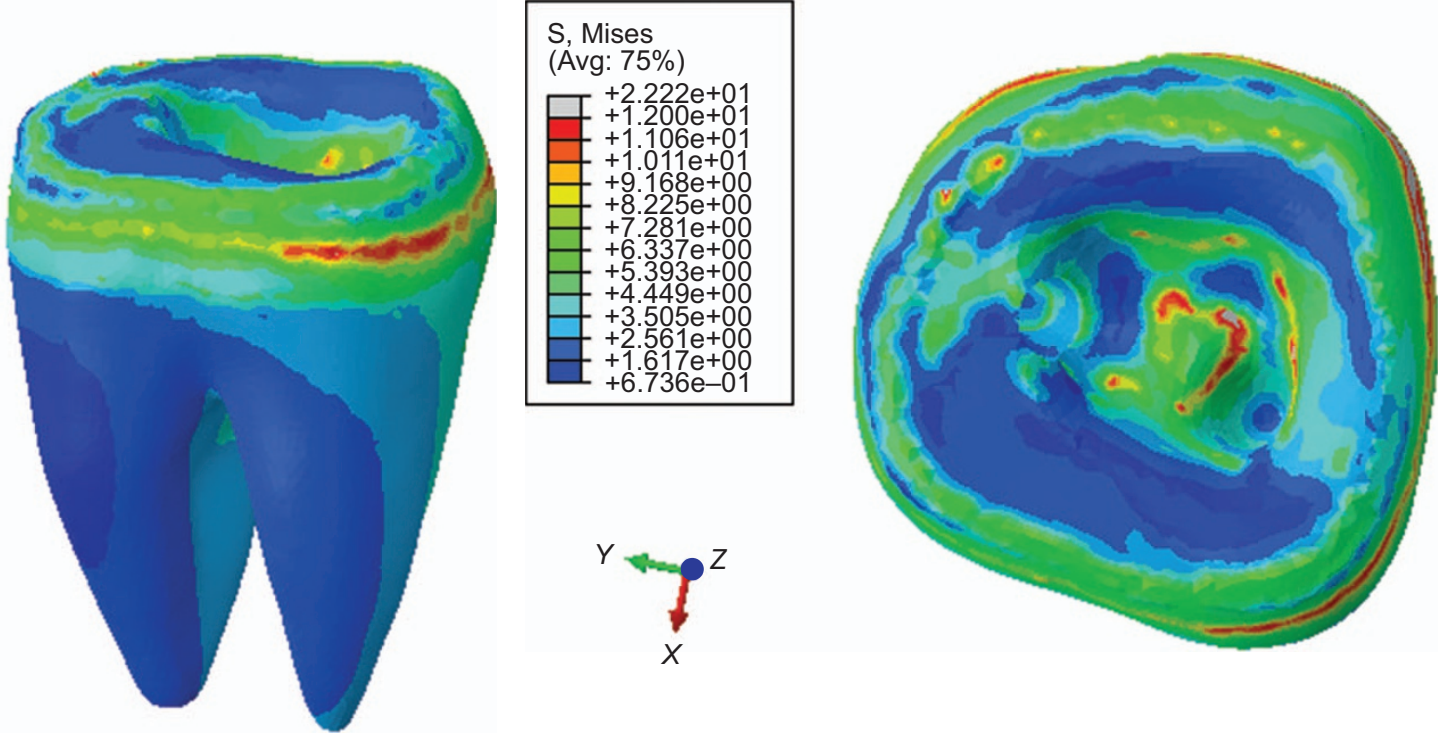

d

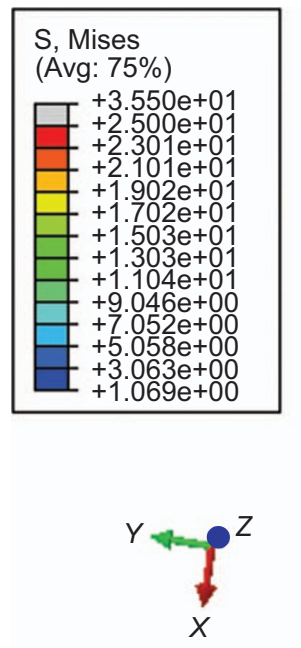

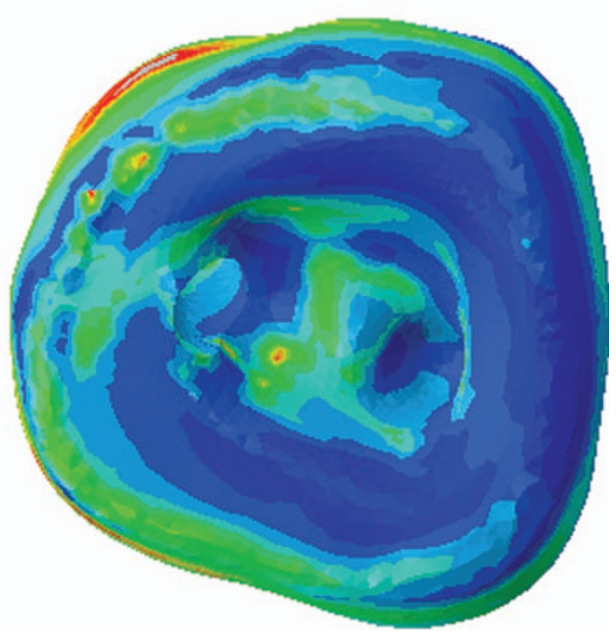

Figure 3 Stress nephogram of the first molar restored using zinc phosphate as adhesive. (a) Stress nephogram of root canal orifice in biting condition; (b) stress nephogram inside the root canal in biting condition; (c) stress nephogram of the root canal in chewing condition; (d) stress nephogram inside root canal in chewing condition.

\section{DISCUSSION}

In this paper, we analyzed the effect of different types of adhesives on multi-piece post-crown restoration solution using finite element analysis. Accurate modeling of adhesives and prosthesis used in our finite element analysis led to more precise results compared with previous studies. ${ }^{15}$ Individual differences could be eliminated because the same first molar root was used for all five assemblies. The experimental error caused by the thickness of different types of adhesives can also be eliminated because the morphologies of the five adhesive models were the same. The stress distributions of the root canal and contact surface between the residual root and prosthesis were the focus of our analysis. However, the experimental methods could not provide the precise data of these positions. Hence, finite elemental analysis was the best tool for dentistry analysis.
The stress distributions of root canals in Table 3 suggest that the stress-shielding effects of different types of adhesives varied. The adhesives with larger Young's modulus transmitted most of the load to the root canal, indicating that these kinds of adhesives cannot protect the root canal effectively. ${ }^{16}$ The impact loads were mostly exerted on the root canal during biting. However, a part of the root canal tissue required to be polished in the course of root canal preparation, resulting in the residual root becoming more vulnerable. Root splitting was another clinical manifestation during the long-term external impact force. In the same loading condition, the adhesives with smaller Young's modulus produced larger deformation that buffered the occlusion impact. This deformation has an important role in protecting the root canal. In the long term, the adhesives with low Young's modulus had greater deformation. The load exerted on the 
a

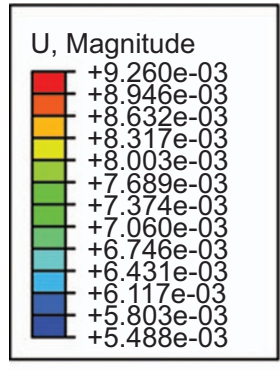

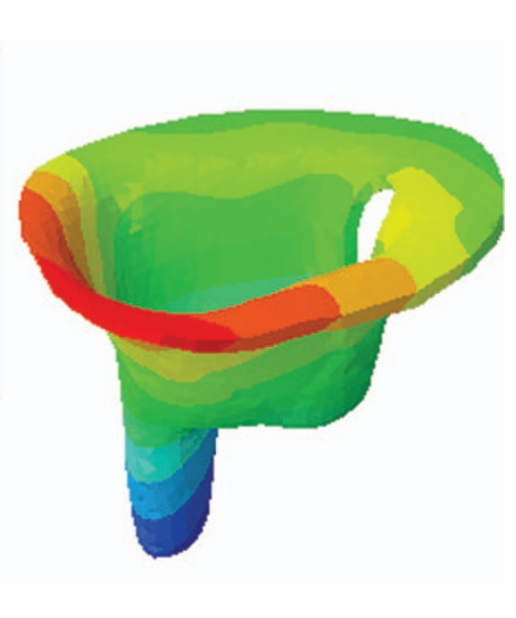
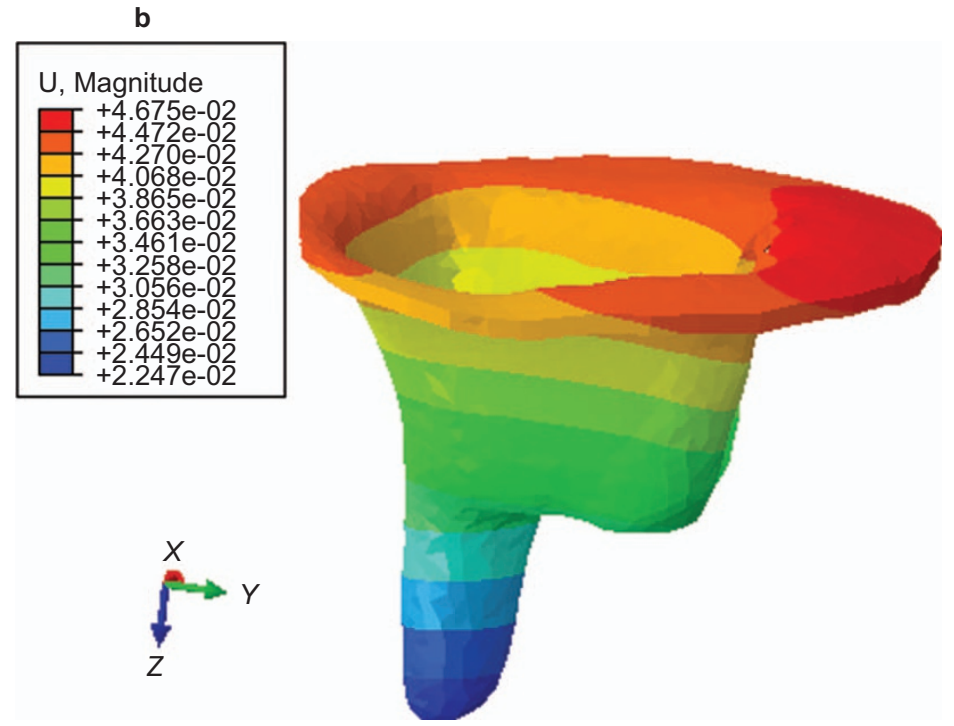

Figure 4 Displacement nephogram of adhesive of zinc phosphate. (a) Displacement of adhesive in biting condition; (b) displacement of adhesive in chewing condition.

root canal orifice was larger. ${ }^{17}$ Stress concentration may be produced in the root canal orifice area while using low Young's modulus adhesive. This process may cause root splitting. The adhesive with large Young's modulus protected the root canal orifice because most of the load was exerted on the root canal.

Deformations of the different types of adhesives varied, but the overall displacement remained the same. ${ }^{18}$ Figure 4 showed that the maximum displacements of all adhesives in biting condition were approximately $0.009 \mathrm{~mm}$. The maximum displacements of all adhesives in the chewing condition were approximately $0.046 \mathrm{~mm}$, which was also shown in Figure 4. The displacements of the five types of adhesives roughly remained the same. The adhesive between prosthesis and root was approximately $0.1 \mathrm{~mm}$. The maximum displacement in the chewing condition can be considered as a large displacement. In the long-term of chewing and occlusion, such a thin adhesive is very brittle and easily broken. Thus, the deformation of the adhesive with low Young's modulus can be recovered after removing the load. Moreover, the deformation of the adhesive with large Young's modulus may break down, resulting in the falling off of the crown. ${ }^{19}$ However, the fracture criteria of all types of adhesives need to be measured in further experiments.

In summary, during the multi-piece post-crown restoration, the protective action of different types of adhesives on the root canal and the root canal orifice were slightly different. Applying adhesives with greater Young's modulus is recommended for patients whose root canal tissues are too thin to avoid root split. These types of adhesives can buffer the impact of long-term chewing and occlusion on the root canal. Adhesives with greater Young's modulus are suggested for patients whose root canal tissues are thick enough. These types of adhesives can reduce the stress concentration on the root canal orifice.

1 Jung $\mathrm{S}$, Min K, Chang $\mathrm{H}$ et al. Microleakage and fracture patterns of teeth restored with different posts under dynamic loading. J Prosthet Dent 2007; 98(4): 270-276.

2 Shimizu A, Hasegawa M, Kishimoto $\mathrm{H}$ et al. An in vitro investigation of the tooth strains associated with four different restorations in class II cavity. J Prosthet Dent 1996; 76(3): 309-314.

3 Darbyshire PA, Messer LB, Douglas WH. Microleakage in class II composite restorations bonded to dentin using thermal and load cycling. J Dent Res 1988, 67(3): 585-587.

4 Ausiello P, Davidson CL, Cascone P et al. Debonding of adhesively restored deep class II MOD restorations after functional loading. Am J Dent 1999; 12(2): 84-88.

5 Fissore B, Nicholls J, Yuodelis RA. Load fatigue of teeth restored by a dentin bonding agent and a posterior composite resin. J Prosthet Dent 1991; 65(1): 80-85.

6 Fan P, Nicholls JI, Kois JC. Load fatigue of five restoration modalities in structurally compromised premolars. Int J Prosthodont 1995; 8(3): 213-220.

7 Torabi K, Fattahi F. Fracture resistance of endodontically treated teeth restored by different FRC posts: an in vitro study. Indian J Dent Res 2009; 20(3): 282-287.

8 Zarone F, Sorrentino R, Apicellar D. Valuation of the biomechanical behavior of maxillary central of maxillary central incisors restored by means of endocrowns compared to a natural tooth: a 3D static linear finite elements analysis. Dent Mater 2006; 22(11): 1035-1044.

9 Asmussen E, Peutzfeldt A, Sahafi A. Finite element analysis of stresses in endodontically treated, dowel-restored teeth. J Prosthet Dent 2005; 94(4): 321-329.

10 Okada D, Miura H, Suzuki C et al. Stress distribution in roots restored with different types of post systems with composite resin. Dent Mater J 2008; 27(4): 605-611.

11 Maceri F, Martignoni M, Vairo G. Mechanical behaviour of endodontic restorations with multiple prefabricated posts: a finite-element approach. J Biomech 2007; 40(11): 2386-2398.

12 Ausiello P, Apicella A, Davidson CL. Effect of adhesive layer properties on stress distribution in composite restorations-a 3D finite element analysis. 2002; 18(4): 295-303.

13 Placido E, Meira J, Lima RG et al. Shear versus micro-shear bond strength test: a finite element stress analysis. Dent Mater 2007; 23(9): 1086-1092.

14 Li LL, Wang ZY, Bai ZC et al. Three-dimensional finite element analysis of weakened roots restored with different cements in combination with titanium alloy posts. Chin Med J 2006; 119(4): 305-311.

15 Su K, Chang C, Chuang S. Biomechanical evaluation of endodontic posts-finite element analysis. J Biomechan 2007; 40(S2): S463-S464.

16 Sorrentino R, Aversa R, Ferro V et al. Three-dimensional finite element analysis of strain and stress distributions in endodontically treated maxillary central incisors restored with different post, core and crown materials. Dent Mater 2007; 23(8) 983-993.

17 Adanir N, Belli S. Stress analysis of a maxillary central incisor restored with different posts. Eur J Dent 2007; 1(2): 67-71.

18 Eskitascioglu G, Belli S, Kalkan M. Evaluation of two post core systems using two different methods. J Endodont 2002; 28(9): 629-633.

19 Nissan J, Dmitry Y, Assif D. The use of reinforced composite resin cement as compensation for reduced post length. J Prosthet Dent 2001; 86(3): 304-308.

(c)

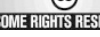

This work is licensed under a Creative Commons Attribution-NonCommercial-NoDerivative Works 3.0 Unported License. To view a copy of this license, visit http:// creativecommons.org/licenses/by-nc-nd/3.0 